\title{
Editorials
}

\section{Airway management: the good, the bad, and the ugly}

Orlando Hung MD FRCPC

A few weeks ago, I responded to an emergency cardiac arrest call. Upon arrival, I was horrified to see a morbidly obese patient gasping for air with ventricular fibrillation displayed on the electrocardiogram monitor. A quick history from the attending house staff confirmed my fear that the patient had a history of difficult intubation. With difficulties in providing adequate bag mask ventilation and with limited available resources, I promptly proceeded with a blind nasal intubation. I was successful in placing an endotracheal tube into the trachea without much difficulty. I breathed a sigh of relief as I wondered what I could have done should this attempt have failed. I believe that most anesthesiologists share the fear of facing an experience like this.

A review of perioperative adverse events related to anesthesia undertaken by the American Society of Anesthesiologists (ASA) in 1990 suggested that many adverse events are related to difficult tracheal intubations. ${ }^{1}$ The ASA has addressed this problem by developing evidence-based practice guidelines for the management of difficult airways (i.e., the ASA difficult airway algorithm). ${ }^{2}$ The goal of these guidelines is to reduce the incidence of adverse events by improving preoperative evaluation and providing clear guidelines and training for difficult laryngoscopic intubation. It should be emphasized that practice guidelines are not intended as standards, but are recommendations to assist the anesthesiologist in the decision-making process. The Difficult Airway Task Force has recommended that these guidelines should be continually revised as warranted by the evolution of medical knowledge, technology, and practice. Recognizing that these guidelines have limitations, the Canadian Airway Focus Group (CAFG) developed strategies for management of unanticipated difficult airway in 1998. ${ }^{3}$ As the current techniques for predicting difficult laryngoscopic intubation are non-specific with low positive predictive values, ${ }^{4}$ the CAFG emphasized the need to equip ourselves with alternative devices and techniques, such as the laryngeal mask airway (LMA), lightwand and rigid fibreoptic laryngoscopes. The CAFG further suggested that training programs should ensure that graduate anesthesiologists are trained in the use of these alternatives. In addition, continuing medical education courses and airway workshops should be available to allow practicing anesthesiologists the opportunity to learn and update their skills with these alternative devices.

In 1998, Rosenblatt et al. ${ }^{5}$ conducted a survey of 472 anesthesiologists in the United States to evaluate their practice patterns in 14 difficult airway case scenarios. Unless a surgical airway was clearly indicated, direct laryngoscopy and fibreoptic-aided tracheal intubation techniques were chosen for most cases by most anesthesiologists. More senior anesthesiologists appeared to favour direct laryngoscopy. In general, there was little use of newer alternative airway devices. The investigators concluded that although the teaching of alternative methods of securing a difficult airway has become more popular, most anesthesiologists rely on direct laryngoscopy and fibreoptic-aided intubation in most clinical circumstances.

In this issue, Jenkins et al. ${ }^{6}$ conducted a similar survey of practicing Canadian anesthesiologists to assess their management of patients with difficult airways. Similar to Rosenblatt's findings, direct laryngoscopy (48\%) and fibreoptic bronchoscopy (34\%) were the two most commonly used techniques for intubation in these difficult airway scenarios. Lighted-stylets, intubating guides, and rigid fibreoptic laryngoscope were

From the Department of Anesthesia, QEII Health Sciences Centre, Halifax, Nova Scotia, Canada.

Address correspondence to: Dr. Orlando Hung, Department of Anesthesia, QEII Health Sciences Centre, Victoria General Site, 1278

Tower Road, Halifax, Nova Scotia B3H 2Y9, Canada. Phone: 902-473-7767; Fax: 902-473-4493; E-mail: hungorla@dal.ca 
rarely used. What is most disturbing about these studies is that the practice of airway management in Canada and in the United States has not changed much during the last decade - even though there have been significant advances in the development of airway techniques. Although one can argue that perhaps it is still too early to observe a change in our practice, there is evidence to show that we have not been successful in the dissemination of new information regarding airway management. Clearly, we must develop strategies to address these critical issues.

Without a doubt, acquisition of airway management skills should begin in our teaching programs. To assess airway management training in Canadian anesthesia residency programs, Dr. Linda Kim and I conducted a survey through the Canadian anesthesia program directors in 1996. At that time, only four programs out of 15 responders had a mandatory airway rotation in their curriculum. All teaching programs had a "difficult airway cart". Apart from laryngoscopic intubation, most programs taught flexible fibreoptic intubation $(100 \%)$ and lightwand intubation (92.9\%). Fewer programs taught rigid fibreoptic laryngoscopic intubating techniques (Bullard laryngoscope - 71\%), intubating guides (71\%), cricothyroidotomy $(71 \%)$, and retrograde intubation $(28.6 \%)$.

In a recent follow-up survey (2002), only four programs (out of 14 responders) have a mandatory airway rotation. All programs now teach flexible fibreoptic intubation, lightwand intubation, intubating guides and LMA. Fastrach (intubating LMA) intubation, an intubating technique introduced in Canada in 2000, has become quite popular (86\%). More centres teach cricothyroidotomy $(86 \%)$ and retrograde intubation $(79 \%)$. However, the use of the rigid fibreoptic laryngoscopy has remained relatively unchanged (64\%). Furthermore, it is my impression that most recent graduates in anesthesia do not have much experience with blind nasal intubation, a technique that is simple and often life-saving.

These surveys have clearly indicated that all Canadian teaching programs recognize the importance of teaching alternative techniques of intubation to residents. Nevertheless, it is disappointing to realize that few programs offer a formal airway rotation as an integral part of resident training. Although most of the new alternative airway techniques can be taught by enthusiastic attending anesthesiology staff on an "ad hoc" basis, it is important to present such basic and critical information in an organized fashion with well designed learning goals and objectives. As the incidence of difficult airway is relatively low, obtaining adequate experience in the use of these techniques for the management of patients with a difficult airway can pose challenges. The use of simulation of difficult airway scenarios is one possible solution. In fact, simulating difficult tracheal intubation has been suggested in the past ${ }^{7,8}$ and this may be particularly relevant in the management of obstetrical patients. ${ }^{9}$

While it is exciting to learn new airway techniques, other key elements of successful intubation cannot be ignored. These include good communication with the patient in the preoperative assessment as well as proper preparation of the patient and equipment. In a letter to the editor in this issue, Dr. Shandling, ${ }^{10}$ a patient, reported his unfortunate experience with his failed tracheal intubation. His complications clearly illustrate the importance of proper preoperative airway examination and good patient communication. It is possible that the attending anesthesiologist did not recognize restricted cervical spine movement during the preoperative airway assessment of Dr. Shandling. It is perhaps more likely that there was a failure in communication between the anesthesiologist and patient, which resulted in a poor outcome.

In summary, it is clear that no single airway assessment can reliably predict a difficult airway. This is due largely to the low incidence of difficult airway, making the positive predictive value of an airway assessment unreasonably low. Nevertheless, preoperative airway assessment is essential as illustrated by Dr. Shandling's letter in this issue of the journal. ${ }^{10}$ It is also critical to equip ourselves with various alternative airway techniques for the unanticipated difficult airway. While there are significant advances in the development of effective airway techniques during the last two decades, most Canadian anesthesiologists do not use these effective alternatives when encountering airway difficulties. Perhaps we should review our training programs and develop strategies in teaching airway management as suggested by the CAFG. Together with well organized airway workshops and regular practice with these alternative techniques in patients with normal airways, it is hoped that all anesthesiologists will acquire and update the necessary airway skills to manage patients presenting with a challenging airway. 


\section{Le contrôle des voies aériennes : du meilleur au pire}

Il y a quelques semaines, j'ai répondu à un appel d'urgence pour arrêt cardiaque. En arrivant sur les lieux, j'ai été consterné de voir un patient souffrant d'obésité morbide, en détresse respiratoire et présentant une fibrillation ventriculaire au moniteur d'électrocardiographie. Un aperçu des antécédents fourni par le médecin traitant en service a confirmé mon appréhension d'une histoire d'intubation difficile. Malgré les difficultés de la ventilation manuelle au masque et au ballon et les limites des ressources disponibles, une intubation nasale à l'aveugle a pu être réalisée rapidement. L'insertion d'un tube endotrachéal dans la trachée s'est faite sans trop de difficulté, à mon plus grand soulagement, mais en pensant à ce qui aurait pu arriver en cas d'échec. La plupart des anesthésiologistes partagent sans doute la crainte d'une telle expérience.

Une revue des incidents indésirables reliés à l'anesthésie, faite en 1990 par l'American Society of Anesthesiologists (ASA), avançait que nombre de ces incidents étaient reliés à des intubations endotrachéales difficiles. ${ }^{1}$ L'ASA s'est attaquée au problème en élaborant des directives cliniques fondées sur des données probantes concernant la conduite à tenir en cas d'intubation difficile (c.-à-d., l'algorithme de l'intubation difficile de l'ASA). ${ }^{2}$ Le but de ces directives est de réduire l'incidence d'événements indésirables en améliorant l'évaluation préopératoire et en présentant des lignes directrices claires et une formation à l'intubation laryngoscopique difficile. Ces directives cliniques ne sont pas des normes, mais plutôt des recommandations pouvant aider l'anesthésiologiste à prendre des décisions. La Difficult Airway Task Force (Groupe de travail sur l'intubation difficile) a recommandé que ces directives soient régulièrement révisées et tiennent compte de l'évolution des connaissances médicales, de la technologie et de la pratique. Les directives ayant leurs limites, un Groupe de consultation canadien sur l'intubation (GCCI) a mis au point, en 1998, des stratégies de gestion de l'intubation difficile imprévue. ${ }^{3}$ Comme les techniques actuelles de prédiction d'une intubation laryngoscopique difficile sont non spécifiques et n'ont que de faibles valeurs prédictives positives, ${ }^{4}$ le GCCI a mis l'accent sur la nécessité de nous doter d'appareils et de techniques de secours, comme le masque laryngé (ML), le stylet lumineux et le fibroscope rigide. Le GCCI a proposé aussi qu'on s'assure que les anesthésiologistes spécialisés reçoivent une formation sur l'usage de ces solutions de rechange. De plus, la formation médicale continue et des ateliers sur l'intubation devraient être offerts pour permettre aux anesthésiologistes de s'initier à ces techniques et de mettre à jour leurs compétences avec ces appareils.

En 1998, Rosenblatt et coll. ${ }^{5}$ ont mené une enquête auprès de 472 anesthésiologistes aux États-Unis afin d'évaluer leurs modèles de pratique dans 14 cas d'intubation difficile. À moins qu'une trachéostomie n'ait été clairement indiquée, la laryngoscopie directe et l'intubation endotrachéale fibroscopique ont été les techniques choisies dans la majorité des cas par la plupart des anesthésiologistes. Les spécialistes plus âgés semblaient favoriser la laryngoscopie directe. En général, on utilisait peu les nouveaux appareils oropharyngés. Les chercheurs ont conclu que, malgré la popularité grandissante de l'enseignement de méthodes nouvelles pour contrer l'intubation difficile en toute sécurité, la plupart des anesthésiologistes comptent encore sur la laryngoscopie directe et l'intubation fibroscopique dans la majorité des cas.

Dans le présent numéro, Jenkins et coll. ${ }^{6}$ ont procédé à une enquête similaire auprès des anesthésiologistes canadiens pour évaluer leur gestion des intubations difficiles. Leurs résultats s'apparentent à ceux de Rosenblatt, la laryngoscopie directe $(48 \%)$ et la fibroscopie bronchique (34\%) étant les deux techniques d'intubation les plus couramment utilisées dans les scénarios d'intubation difficile présentés. Les stylets lumineux, les guides d'intubation et le laryngoscope fibroscopique rigide sont rarement utilisés. Fait plus troublant encore, la façon d'aborder les difficultés d'intubation n'a pas vraiment changé au Canada et aux États-Unis pendant la dernière décennie, même s'il y a eu des progrès significatifs des techniques d'intubation. Certains diront qu'il est peut-être trop tôt pour observer un changement de pratique, mais il semble évident que nous n'avons pas réussi à diffuser les nouvelles informations concernant le contrôle des voies respiratoires. Nous devons élaborer des stratégies pour traiter de ces importantes questions.

L'acquisition d'habiletés en assistance respiratoire doit commencer, sans aucun doute, dans nos programmes de formation. Pour évaluer la formation en assistance respiratoire des programmes canadiens de résidence en anesthésie, le Dr Linda Kim et moi-même avons mené une enquête pancanadienne auprès des directeurs de programmes d'anesthésie en 1996. À ce 
moment-là, parmi 15 programmes, seulement quatre présentaient une rotation obligatoire en intubation. Tous les programmes d'enseignement possédaient un "chariot pour intubation difficile". Mis à part l'intubation laryngoscopique, la plupart des programmes enseignaient l'intubation fibroscopique flexible (100\%) et l'intubation avec stylet lumineux $(92,9 \%)$. Peu de programmes présentaient l'intubation fibroscopique rigide (71 \% pour le laryngoscope Bullard), les guides d'intubation (71 \%), la cricothyroïdotomie (71 \%) et l'intubation rétrograde $(28,6 \%)$.

Dans une récente enquête de suivi (2002), quatre programmes seulement (sur 14) avaient une rotation obligatoire sur l'intubation. Tous les programmes enseignent maintenant l'intubation fibroscopique flexible, l'intubation avec stylet lumineux et présentent les guides d'intubation et les ML. L'intubation Fastrach (ML d'intubation), une technique introduite au Canada en 2000, est devenue assez populaire (86 $\%)$. Dans un plus grand nombre de centres, on enseigne maintenant la cricothyroïdotomie $(86 \%)$ et l'intubation rétrograde (79\%). Cependant, l'usage de la laryngoscopie fibroscopique rigide est demeuré relativement stable $(64 \%)$. En outre, les derniers diplômés en anesthésie ne semblent pas avoir beaucoup expérimenté l'intubation nasale à l'aveugle, une technique simple qui peut souvent sauver une vie.

Ces enquêtes ont clairement indiqué que tous les programmes d'enseignement canadiens reconnaissent l'importance d'enseigner des techniques d'intubation de remplacement. Néanmoins, il est malheureux de constater que peu de programmes offrent une rotation formelle en intubation comme partie intégrante de la résidence. Bien que la plupart des nouvelles techniques puissent être enseignées sur une base "expérimentale" par un personnel d'anesthésiologie enthousiaste, il faut que ces informations fondamentales et critiques soient présentées de manière organisée et selon des buts et des objectifs de formation bien définis. Comme l'incidence d'intubation difficile est relativement faible, l'apprentissage d'une expérience suffisante des nouvelles techniques peut constituer tout un défi. L'usage de la simulation de scénarios d'intubation difficile apparaît donc comme une solution possible. De fait, la simulation d'intubation endotrachéale difficile a déjà été évoquée ${ }^{7,8}$ et peut être particulièrement pertinente auprès des patientes d'obstétrique. ${ }^{9}$

S'il est enthousiasmant d'apprendre de nouvelles techniques d'intubation, il ne faut pas oublier d'autres éléments clés d'une intubation réussie. Nous pensons à une bonne communication avec le patient pendant l'évaluation préopératoire ainsi qu'à la préparation appropriée du patient et du matériel. Dans une lettre publiée dans le présent numéro, le Dr Shandling, ${ }^{10}$ un patient, rend compte de son expérience déplorable d'une intubation endotrachéale non réussie. Les complications rapportées illustrent clairement l'importance d'un examen préopératoire compétent des voies respiratoires et d'une bonne communication avec le patient. Il est possible que l'anesthésiologiste traitant n'ait pas constaté le mouvement restreint de la colonne cervicale pendant l'évaluation préopératoire du Dr Shandling. Un manque de communication entre le médecin et son patient semble plus probable, ce qui a entraîné des complications.

En résumé, il est clair qu'une évaluation des voies respiratoires ne peut permettre, à elle seule, de prédire des difficultés d'intubation de façon fiable. C'est en grande partie à cause de la faible incidence d'intubation difficile, ce qui réduit considérablement la valeur prédictive positive de l'examen des voies aériennes. L'évaluation préopératoire des voies respiratoires est pourtant essentielle comme le montre la lettre du Dr Shandling. ${ }^{10}$ Nous devons aussi nous doter de diverses techniques d'intubation de remplacement en cas d'intubation difficile imprévue. Même si les techniques d'intubation efficaces ont connu des progrès significatifs pendant la dernière décennie, la majorité des anesthésiologistes canadiens n'utilisent pas encore ces solutions de rechange en cas d'intubation difficile. Peut-être faut-il revoir nos programmes de formation et élaborer des stratégies d'enseignement de l'assistance respiratoire comme le suggère le GCCI. Des ateliers bien organisés sur l'intubation et une pratique régulière des techniques de remplacement chez des patients dont les voies aériennes sont normales pourraient, nous l'espérons, permettre à tous les anesthésiologistes d'acquérir et de mettre à jour les compétences nécessaires pour faire face aux difficultés d'intubation.

\section{References}

1 Caplan RA, Posner KL, Ward RJ, Cheney FW. Adverse respiratory events in anesthesia: a closed claims analysis. Anesthesiology 1990; 72: 828-33.

2 Practice guidelines for management of the difficult airway. A report by the American Society of Anesthesiologists Task Force on Management of the Difficult Airway. Anesthesiology 1993; 78: 597-602.

3 Crosby ET, Cooper RM, Douglas MJ, et al. The unanticipated difficult airway with recommendations for management. Can J Anaesth 1998; 45: 757-76.

4 Yentis SM. Predicting difficult intubation-worthwhile exercise or pointless ritual? Anaesthesia 2002; 57: 105-9. 
5 Rosenblatt WH, Wagner PJ, Ovassapian A, Kain ZN. Practice patterns in managing the difficult airway by anesthesiologists in the United States. Anesth Analg 1998; 87: 153-7.

6 Jenkins K, Wong D, Correa R. Management choices for the difficult airway by anesthesiologists in Canada. Can J Anesth 2002; 49: 850-6.

7 MacQuarrie K, Hung OR, Law A. Tracheal intubation using a Bullard laryngoscope for patients with a simulated difficult airway. Can J Anesth 1999; 46: 760-5.

8 Ovassapian A, Yelich SJ, Dykes MH, Golman ME.

Learning fibreoptic intubation: use of simulators v. traditional teaching. Br J Anaesth 1988; 61: 217-20.

9 Cormack RS, Lehane J. Difficult tracheal intubation in obstetrics. Anaesthesia 1984; 39: 1105-11.

10 Shandling $B$. Decreased neck mobility and postoperative complications (Letter). Can J Anesth 2002; 49: 888. 